\title{
Polarized vernier optometer
}

\author{
NICHOLAS M. SIMONELLI \\ University of Illinois at Urbana-Champaign, Urbana, Illinois 61801
}

\begin{abstract}
An optometer that makes use of polarized light and is based on the Scheiner principle is described. It is similar in construction and use to the better-known laser optometer, but it is less expensive, avoids some of the problems inherent in using lasers, and is responded to very well by subjects in behavioral research. Uses of the device are discussed, and the laser and vernier optometers are contrasted. It is concluded that the polarized vernier optometer is an economical and effective alternative to the laser optometer in behavioral research settings.
\end{abstract}

Research has increased in recent years on both the effects of accommodation on psychophysical and perceptual phenomena and the "dark focus," or "intermediate resting position," of accommodation (see Leibowitz \& Owens, 1978). This is in large part due to greater availability of measuring devices. The infrared optometer and eye tracker (Crane \& Steele, 1978) have proved to be useful devices for the continuous, objective measurement of refractive state, but they are complex and very expensive. The laser optometer (Hennessy \& Leibowitz, 1972) is a simpler device that yields discrete measures of refractive state; it is being employed in a number of laboratories. It is relatively easy to construct, straightforward to use, and is much lower in cost.

Recently, another optometer has been developed that is very simple to construct and is less expensive than the laser optometer. The unit uses polarized light and is suitable for use in many research situations.

\section{PRINCIPLE}

Moses (1971) briefly described an optometer principle that takes advantage of the properties of polarized light. Figure 1 is an illustration of this principle. Using two pairs of perpendicularly oriented polarizing filters, the retinal image of a viewed object (in this case, a horizontal bar) splits when the retina is not conjugate with the plane of that bar. Likewise, the image is whole when the retina is conjugate with the bar. This is an application of the Scheiner principle (see Duke-Elder, 1970, p. 155) whereby one image (here, one half of the bar) is directed through the upper half of the pupil

The author is now with the Copying Products Division, 3M Technical Service, Building 234-1F-12, 3M Center, St. Paul, Minnesota 55144. This work was conducted under Air Force Office of Scientific Research Contract USAF F49620-77-C-0017; Alfred R. Fregley is the scientific monitor. Many thanks are extended to Robert T. Hennessy for his initial demonstration of the polarized vernier phenomenon, his invaluable aid in the development of the optometer, and his comments on the manuscript. Thanks are also extended to Robert A. Moses for his advice and comments and to R. Annette Sedlund for typing the manuscript.
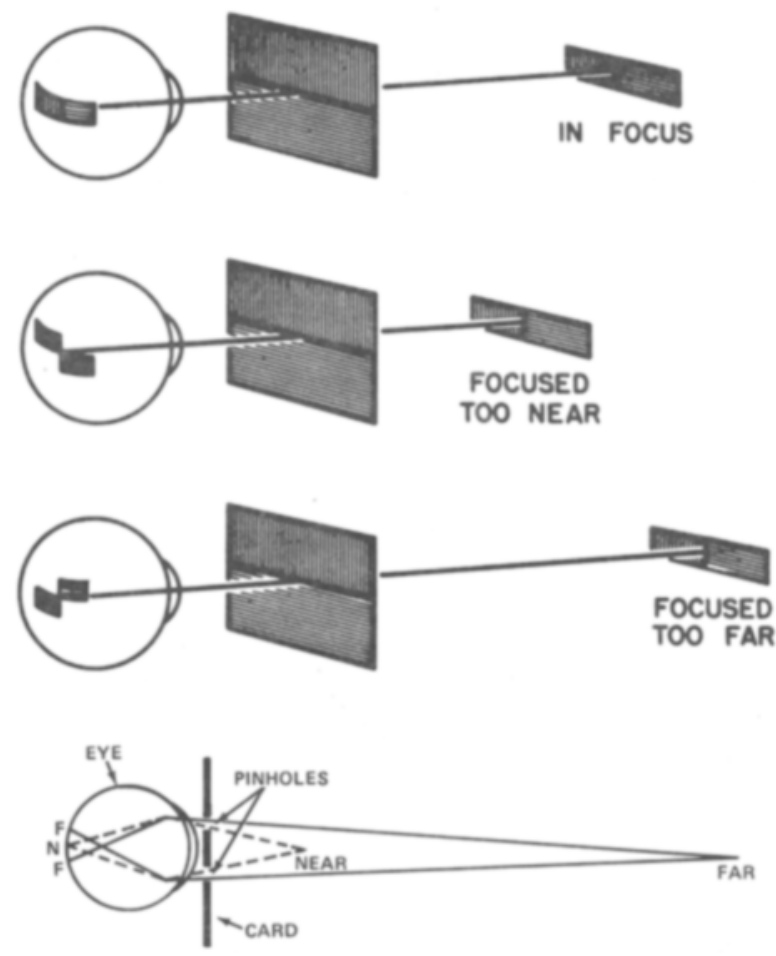

Figure 1. Top: Illustration of the polarizing phenomenon (adapted from Moses, 1971), demonstrating the retinal images created for three states of focus. Bottom: More detailed illustration of the Scheiner principle. The eye is focused near. Thus, the two distant rays do not converge on the retina but are displaced relative to each other, as with the retinal ("bar") images above. The polarized filters in the optometer function as the pinholes.

and another image (the other bar half) is directed through the lower half.

This direction of bar halves through different portions of the pupil is accomplished by creating barsegment images whose light rays are of different polarities (indicated in the figure by the direction of the parallel lines in the filters). The left half of the target bar, for instance, is vertically polarized. Such rays will pass through the upper portion of the next pair of filters (with some absorption loss), as the polarities of 
the light and filter are identical. These vertical rays, however, cannot pass through the horizontal filter below.

Consequently, when this second pair of filters is aligned to "split" the pupil in half, the vertically polarized rays from the left portion of the target bar enter only the upper half of the pupil. Similarly, the image of the right half of the bar enters only the lower half of the pupil. When the eye is focused on the bar, both halves "meet" at the retina and reform the whole bar. Moreover, one half shifts relative to the other when the eye is focused in front of or behind the stimulus bar. The amount and direction of the shift are related to the amount and direction of the focal error.

Thus, if a viewer reports alignment of the two bars, his or her accommodative state is correct for the distance from the eye to the stimulus bar. The viewer's report of the direction of misalignment indicates the direction of the focal error. The use of such a split bar, or vernier, yields the device's name: polarized vemier optometer. Although the vernier effect is relatively straightforward and easy to obtain, no reports of research involving the use of a simple refracting device using this phenomenon have been found.

Allen (1949) used polarized images as a calibration in a more complex arrangement to photograph the third Purkinje image at high speed. His device was for measuring accommodation more or less continuously. Biersdorf (1960) used a polarized vernier in a similar manner. In both cases, the basic accommodation measurement was from the analysis of photographs. Here, however, the measurement is taken from the observer's verbal responses to the vernier itself.

\section{COMPONENTS OF THE OPTOMETER}

Figure 2 illustrates the principal components of the optometer. The polarizing aperture and the box producing the polarized vernier create the split bars used in the measurement of refractive state. Adding a shutter

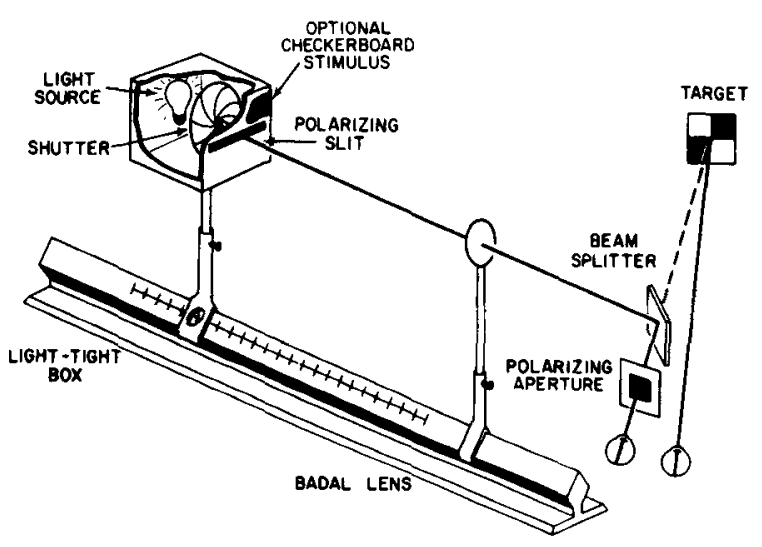

Figure 2. Schematic of the polarized vernier optometer. to the system allows the brief exposures needed to employ the "bracketing technique" commonly used in laser refraction. That is, refractive state is measured using repeated short stimulus exposures, each adjusted according to the observer's response to the previous stimulus, until the correct response is found. This technique enables measurement of the dark focus and, with appropriate combining glass, superposing of the vernier onto another scene or experimental stimulus. With the shutter open, the vernier can be viewed continuously for measurements such as the far point (the farthest point to which the eye can focus).

The use of a box enclosure as shown in Figure 2 is optional for room-lighted situations. However, for measuring the dark focus, light scattering can be avoided by enclosing the light source, which, as illustrated, is a $40-\mathrm{W}$ incandescent bulb. A shutter provides the only means for light to escape the box. The light then passes through the polarizing slit. The observer's eye is placed at the posterior focal plane of a convex lens, and the bar is moved relative to the anterior focal plane, varying its dioptric power. (This arrangement is known as a Badal optometer.) The distance from the polarizing slit to the center of the lens determines the focal power of the bar images.

The exact power of the images, when the polarizing slit is at a specific position from the lens, is given by the equation $Q=F^{2} u-F$, where $Q$ is the power of the image in diopters, $\mathrm{F}$ is the power of the lens in diopters, and $u$ is the distance of the polarizing slit from the lens in meters. (Note that the sign of Q will be the opposite of what one would expect, due to previous conventions in optics. A $Q$ of -4 , for example, means $+4 \mathrm{D}$ of power.) The current optometer used by the author contains a +8.0-diopter (D) lens $(F=.125 \mathrm{~m})$, requiring only $12.5 \mathrm{~cm}$ of movement to place the bar at from 0 to just under $8 \mathrm{D}$. The use of a convex lens also allows positioning of the light box.

The observer should not wear contact lenses if this can be arranged. With each blink, the lens may float over the surface of the cornea and cause distortions as the lens floats back into position. The observer may see shifts in the bars as the lens moves.

\section{USES}

Measurement of the dark focus is accomplished using a procedure identical to that employed with the laser optometer. After a brief exposure of the vernier, the observer reports the relative positions of the two bars and the experimenter adjusts the position of the box forward or backward as necessary. When the observer reports the bars aligned (by responding "even"), the experimenter has located the point conjugate with the observer's retina. Of course, this point is actually a range, over which the "even" response will be given. The size of this range is partially a function of the pupil 
width. The wider the pupil, the smaller the range will be.

This can be seen in Figure 3, where the distance " $d$ " is the minimum displacement between the two bars necessary to see them as not aligned. With a narrow pupil, a greater amount of accommodative change can occur before $d$ is reached. A wide pupil admits more of the peripheral rays, which are imaged farthest apart on the retina.

Vernier acuity is quite sensitive, and, if the polarizing aperture is properly positioned, most observers notice a shift in the bars when the vernier is at the edge of the neutral zone and is moved by .1 D. Smaller pupils yield a wider neutral zone, but even this is usually only .2-.6 D. It is convenient to choose one bar, the left, for instance, and have the observer always report its position relative to the other bar ("higher," "lower," or "even"). As the bars near alignment, they come into sharper focus. Conversely, as the bars move apart, they are increasingly out of focus, but the discrimination is unimpaired as the separation between the bars is greater.

An astigmatism in the vertical meridian of the eye will manifest itself as bars seen to be of different widths. That is, if the upper half of the cornea refracts at a power different from that of the lower half, both bars will not be in focus on the retina at the same time. When one bar is in focus, the other will be blurred and therefore slightly wider. In such a case, the measurement obtained is not exact for the entire corneal surface, but only for part of it. (Of course, any single axial measurement of refractive error for an astigmatic eye, no matter the method, is only approximate.)

The device can also be used to measure the near and far points of the eye. Theoretically, an observer can focus on the vernier, seeing it aligned, and follow it as it is moved away from his eye out to his far point, at which time the vernier will break (the halves will not be aligned). The observer will be unable to accommodate farther outward to align the bars. A similar procedure could be followed for the near point, following the vernier inward until it can no longer be held together.

In practice, however, it has been found that microfluctuations of accommodative state, which are easily
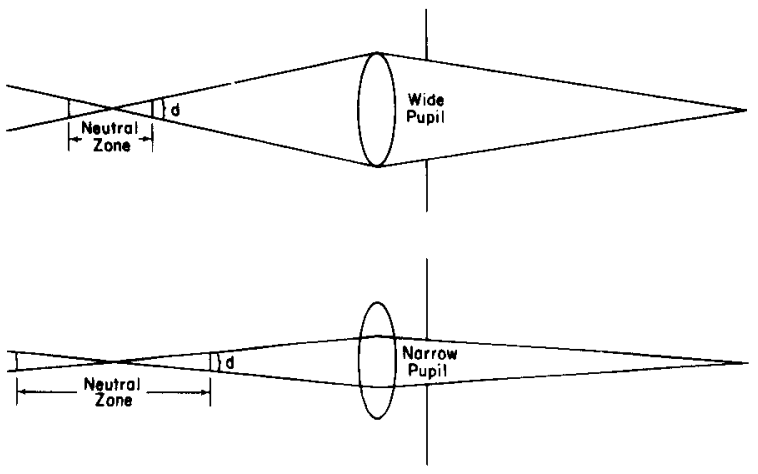

Figure 3. Relationship between pupil width and neutral zone. reflected in the sensitive vernier, give rise to false break points with untrained participants. For example, when following the vernier out to the far point, a sudden, small accommodative shift inward causes a shift in the bars identical to that observed when the far point is reached. Several such false far points may be observed during one measurement.

Moreover, most people usually do not focus accurately on a near target, but rather a bit farther out (see Sheard, 1922 , p. 93), and therefore the vernier may not appear aligned when the observer is looking at it and has it "in focus." A satisfactory procedure has been to place a well lit checkerboard pattern in the plane of the vernier (as seen in Figure 2) and approach the far point from beyond ("out to in") rather than from within. The observer will report initial misalignment (as it is too far out for his eye to focus on), and as the vernier and checkerboard slowly approach the far point, they will be increasingly clear. At the far point, the checkerboard is in focus and the vernier is aligned.

In measuring the near point, a similar procedure is unsatisfactory. An observer's "near point" is largely a function of the amount of convergence (with associated discomfort) that he is willing to exert. Moving a nearpoint card toward the observer's face, as an optometrist does, gives an approximation of an observer's near point. However, the level of tolerable discomfort varies among and within observers, which varies the near point measured. Thus, with young observers, a monocular view of the vernier, requiring no convergence for fusion of two images, usually yields a near point several diopters farther out than a binocular near point, which is subject to individual differences in blur interpretation.

The accommodative responses to other targets can be measured by the brief-exposure technique. Viewing both the vernier and a target simultaneously is accomplished with a beam splitter, as seen in Figure 2. This procedure is used to measure accommodative response to virtually any target and is identical to that used with the laser optometer. A variable power supply to the light allows vernier brightness to be adjusted appropriately for the target. An exposure time for the vernier of $250 \mathrm{msec}$ has been found to be quite satisfactory for most observers. The exposure time must be less than the reaction time for visual accommodation $(300-400 \mathrm{msec})$ so that the lens has no opportunity to accommodate to the vernier rather than to the target.

\section{COMPARISON WITH LASER REFRACTION}

The simplicity of the polarized vernier optometer results in several differences with the laser optometer that may make the vernier optometer preferable. The laser speckles do not stimulate accommodation (one of the virtues of the device), but "Newton rings" produced by some of the lenses can induce accommodation. If exposure time is too long, an observer's accommodation 
may be drawn toward the rings, interfering with his response. Eliminating the rings requires a spatial filter, which is not always available in the less expensive laser assemblies. There are occasional observers who cannot readily interpret the speckles. These few people are unable to give meaningful, confident reports of the speckle movement and are rejected as observers from research using laser refraction. No observer in the author's experience, however, has reported that he could not interpret the vernier stimulus.

A structural advantage of the polarizing technique is that the plane of the vernier can be brought right up to the lens, allowing production of a dioptric power very near the power of the lens. That is, little range is lost. The typical laser arrangement incorporates a moving mirror that limits the maximum accommodation that can be measured to well short of the power of the lens used, although newer designs have avoided this problem. The reduced measurement range is due in part to the fact that the "plane of stationarity" in laser refraction is actually behind (not on) the drum surface (Charman, 1974) and in part to the mirror's forward position. Thus, for a given lens, which determines the proximity of the device to the observer, a larger range of accommodation can be measured with the vernier arrangement. Additionally, the maintenance of a light bulb is cheaper than that of a laser.

Eye position is a critical factor that can be a problem for both optometers. With both devices, the researcher wants the observer's eye at the focal point of the lens. However, slight changes of head position vertically do not noticeably impair the observer's view of the speckles. With the polarizing optometer, on the other hand, the polarizing aperture must split the pupil, and vertical head movements are more detrimental. In general, the same type of head restraint used in laser refraction is adequate, but, in particular, the use of a headrest and chinrest combination has proved very effective. Proper use of the headrest keeps head movements minimal for most observers, although there are those who have significant difficulty in this respect. A possible improvement is to incorporate the polarizing aperture into an eye patch or similar eye covering. With this arrangement, head movements should not disturb the positioning of the polarizing aperture with respect to the pupil.

In an experimental comparison of the two optometers (Simonelli, 1979), measurements of the dark focus obtained with each device were in very close agreement.
(A nonreliable difference of .16 D was found.) Additionally, successive measurements taken with the vernier showed greater agreement than those taken with the laser. That is, there was more intraobserver variability from one measurement to the next in the laser measurements than in the vernier measurements. Subjects were also asked to evaluate the ease with which they could make responses to the stimuli. The observers rated the vernier as easier to respond to and indicated more confidence in their vernier responses.

\section{SUMMARY}

The polarized vernier optometer is an economical and effective alternative to the laser optometer in behavioral research settings. The device may allow investigators who would otherwise not have access to a refracting device to measure refractive state in psychophysical research.

\section{REFERENCES}

Allen, M. J. An objective high speed photographic technique for simultaneously recording changes in accommodation and convergence. American Journal of Optometry, 1949, 26, 279-289.

Biersdorf, W. R. The utility of the anterior lens Purkinje image as a measure of accommodation. American Journal of Optometry, 1960, 37, 352-362.

Charman, W. On the true position of the plane of stationarity in laser refraction. American Journal of Optometry and Physiological Optics, 1974, 51, 832-837.

Crane, H. D., \& Steele, C. M. Accurate three-dimensional eye tracker. Applied Optics, 1978, 17, 691-705.

Duke-Elder, S. System of ophthalmology (Vol. 5). St. Louis: Mosby, 1970.

Hennessy, R. T., \& Leibowitz, H. W. Laser optometer incorporating the Badal principle. Behavior Research Methods \& Instrumentation, 1972, 4, 237-239.

Leibowitz, H. W., \& Owens, D. A. New evidence for the intermediate position of relaxed accommodation. Documenta Ophthalmologica, 1978, 46, 133-147.

Moses, R. A. Vernier optometer. Journal of the Optical Society of America, 1971, 61, 1539.

ShEARD, C. The comparative value of various methods and practices in skiametry. American Journal of Physiological Optics, 1922, 3, 177-208.

Simonelli, N. M. The dark focus of visual accommodation: Its existence, measurement, and effects. Unpublished doctoral dissertation, University of Illinois at Urbana-Champaign, 1979.

(Received for publication October 23, 1979; revision accepted February 11,1980 .) 